\title{
Intracranial cannulation of small animals
}

\author{
ROBERT M. GLEESON, MICHAEL G. DRAGUNOW, NEIL F. KIRTON, \\ JOHN W. VILLIGER and DOUGLAS L. CHUTE \\ University of Otago, Dunedin, New Zealand
}

\begin{abstract}
Special difficulties are encountered when attempting to provide intracranial cannulation of small animals that have floating or fragile skull bones. The technique described was developed for mice but seems applicable to other species. The cannula system is inexpensive and easy to use. A wound clip anchor seems superior to either skull screws or bonded acrylic, producing less damage to the brain and no necrosis of bone or tissue on the wound margin. Patency data up to 25 days is presented, and the system is as efficacious as other techniques.
\end{abstract}

Many aspects of neuropharmacological research require an inexpensive and reliable method for repeated administration of chemical substances into selected brain regions of unanesthetized animals. A number of elegant cannulation techniques have been developed for implantation in rats (Crane \& Glick, 1979; Laird, Hermanson, \& Huxtable, 1979; Routtenberg, 1972). The implantation of cannulas in mice is particularly difficult because of the gracility of the skull and the small cranial area available for anchoring the cannula. These factors generally preclude the use of the techniques devised for cannulating rats. Kokkinidis, Raffler, and Anisman (1977) attempted to overcome the anchoring problems by using stainless steel jewelers' screws with a hole drilled through the center and a 26-gauge needle section soldered into the hole. Anchoring was obtained by four rotations of the screw in a predrilled skull hole, and the screw and surrounding skull area were then coated with dental acrylic cement.

When dental acrylic cement is used to prevent lateral movement of a cannula, the cement must be firmly bound to the skull, and this can only be achieved if the bone is thoroughly prepared by the application of a polar lipid solvent (e.g., xylene). This results in the danger of tissue destruction around the wound margin, due to the action of the lipid solvent and necrosis of the bone of the skull, and potentially to the brain itself, by penetration of the dental acrylic solvent.

The technique described here avoids these difficulties by using a "Michele" wound clip as the anchoring mechanism. Although dental acrylic is used as a bridge between the cannula and the wound clip, the acrylic need not be firmly bound to the bone of the skull. Accordingly, the periostium can be left intact, obviating the usual problems with the use of acrylic. Anchoring skull screws can be used, but the thinness of the mouse skull and the high risk of cerebral lesion make them less than satisfactory. Using solder risks fluid

Send reprint request to Douglas L. Chute, Department of Psychology, University of Otago, Dunedin, New Zealand. This research was supported in part by Otago Research Committee Grant 37885. contact, which would permit toxic substances access to the brain.

The following is applicable to any cannulation in which the bones are fragile or floating as in birds, fish, mice, or juveniles of other species.

\section{METHOD}

\section{Cannula Construction}

Each guide cannula is fashioned from an 18-gauge hypodermic needle with a polypropylene "Luer lock" hub. The hub is removed, and the shaft of the needle is roughly cut to a length of approximately $1.5 \mathrm{~mm}$. The shaft is then reduced to a length of $1 \mathrm{~mm}$, with the tip rounded and smoothed with an oilstone. Shaping and burr removing should be checked periodically by examining the cannula tip under a binocular microscope. The purpose of a cannula of such short length is to act solely as a guide for a microsyringe. Because of the cannula's short length, cortical damage will be minimized. The external portions of the guide cannula maintain the accuracy of the injection angle, and, if desired, the needle hub can be left attached to provide the Luer lock for a firm syringe fitting. Although the experimental requirements for which this system was devised (Villiger \& Chute, 1979) involved a large guide cannula, the ideal would be one as small as possible, 27 gauge or smaller to reduce the risk of lesioning the cortex.

Our research required an intracranial injection into the lateral ventricle of the mouse brain. National Institutes of Health NZ/A mice (average weight $22 \mathrm{~g}$ ) were anesthetized with Fluorothane $\mathbf{R}$ (halothane B.P., Imperial Chemical Industries, Ltd., Great Britain).

\section{Cannula Placement and Anchoring}

A longitudinal incision of approximately $1.5 \mathrm{~cm}$ is made in the scalp slightly off midline. A set of dividers is used to mark a point on the skull $2 \mathrm{~mm}$ rostral to lambda and $2 \mathrm{~mm}$ to the right (or left) of the sagittal suture. An 18-gauge hole is hand drilled at this point with a drill bit mounted in a pin vise and sleeved with polyethylene tubing to prevent penetration of the dura mater. A $14-\mathrm{mm}$ stainless steel Michele wound clip is clamped laterally across the skull so that the points of the clip penetrate the temporal muscle and lodge below the dorsolateral flexure. Better control over the "snap action" of the clip is afforded by cutting a small indentation in the clip at its midpoint before use. The cannula is then placed in the skull hole, and dental acrylic cement is molded around it and the wound clip to form a bridge between the cannula and its anchor. When the acrylic is dry, a rubber plug (the tip from the plunger of a 1-cc syringe) is placed over the end of the cannula to prevent seepage of cerebrospinal fluid and to maintain patency of the cannula.

To inject a substance, the rubber plug is cut away and a 


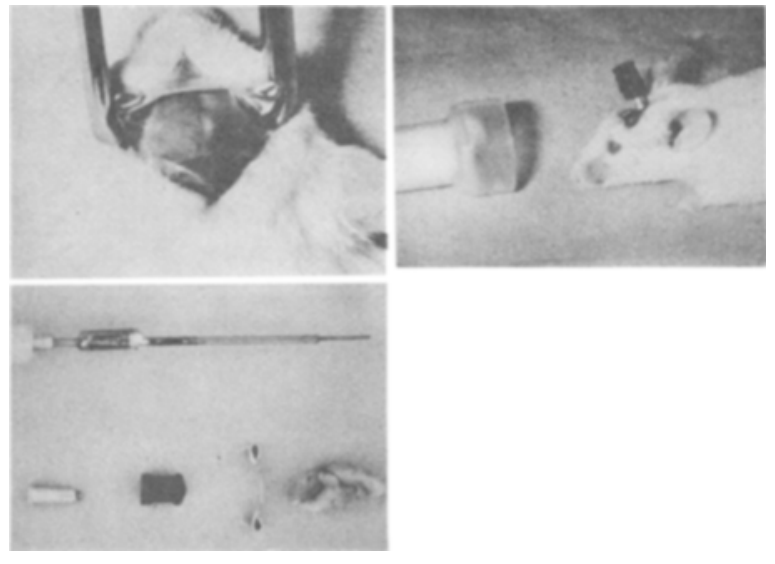

Figure 1. Top left: Placement of the Michele wound clip that anchors the cannula to the skull. Top right: Ether anesthesia is maintained by a "nose cone," the cartiridge of a 3-cc disposable syringe. Wound clip and cannula assembly are shown in place prior to the application of dental acrylic. Bottom left: Shown are a microsyringe with adjustable sheath to control depth of injection, a guide cannula constructed from a disposable needle, the cannula cap (formerly the rubber plunger from a $1-\mathrm{cc}$ syringe), a 14-mm Michele clip, and the left hemisphere of a mouse brain, showing methylene blue staining of ventricles.

microsyringe, with a depth collar fitted to the needle shaft, is inserted into the cannula. With appropriate adjustments for depth, the needle can be inserted directly through the rubber plug when multiple injections are indicated. If the Luer lock hub is left on the cannula, the rubber plug fits snugly inside and is easy to remove or pierce at the time of injection. Such a taller structure on the head is, however, a greater risk, with concomitant decrease in patency over time (Figure 1).

\section{RESULTS}

\section{Patency}

We recorded the percentage of mice with the cannula still intact on the skull and patent at 9 and 16 days postsurgery for one group of animals $(\mathrm{N}=32)$ and at 9,16 , and 25 days for a second group $(\mathrm{N}=62)$. All mice retained patent cannulas for at least 16 days following surgery. This decreased to $53 \%$ after a 25 -day period (Figure 2).

\section{Accuracy}

The accuracy of the cannulation technique was evaluated by injecting methylene blue stain into the lateral ventricles, via the cannula, of living mice and then examining the spread of the stain in the mice brains $5 \mathrm{~min}$ after decapitation. The percentage with stain directly in the lateral ventricle was approximately $82 \%$ at 9,16 , and 25 days postsurgery (Figure 3 ). These results appear to compare favorably with those achieved by other techniques.

During necropsy, the cannula and injection track in the brain was observed and related to stereotaxic coordinates (Lehmann, 1974) that yielded average coordinates of $\mathrm{AP}=3.5 \quad(\mathrm{SD}=.2)$, lateral $=2.4 \quad(\mathrm{SD}=.2)$. Some wound clip damage was also noted, with the following average coordinate locations on the cerebral cortex: right wound clip damage $-\mathrm{AP}=3.7(\mathrm{SD}=.5)$, lateral = $4.0(\mathrm{SD}=.17)$, and vertical $=3.2(\mathrm{SD}=.4)$; left wound clip damage $-\mathrm{AP}=3.7(\mathrm{SD}=.5)$, lateral $=3.9(\mathrm{SD}=.3)$, and vertical $=3.2(\mathrm{SD}=.4)$.

With a suitable adaptation to an electrode carrier, stereotaxic placement of the guide cannula is reasonably simple. For intracerebroventricular use, however, we simply place the cannula in relation to skull coordinates. Considerable time is saved, and potential damage to the animal is avoided because no head holder is necessary and anesthetic risk is reduced. Inaccuracy, indicated by the stain, is, for the most part, due to both excessive depth and caudal angling, resulting in subdural staining of the brain stem.

Damage from wound clips, where it exists, is minor and usually is caused by compression of the skull, which seldom results in gliosis of cerebral tissue.

\section{DISCUSSION}

Generally, the mice, when examined, were in good health and showed no obvious deleterious effects from cannulation. The cannulas were lightly packed with tissue, and there was some dried serum under the skull caps. The skull bone was in good condition with no indication of necrosis, but a slight thickening of the periostium immediately under the wound clip was

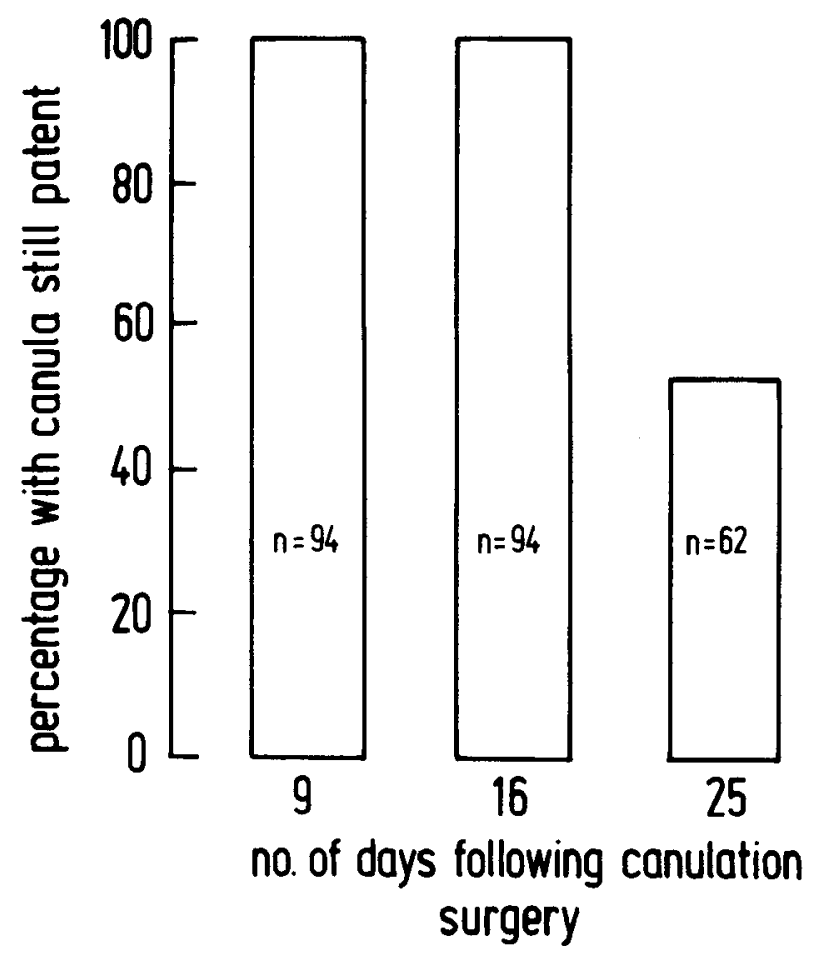

Figure 2. The wound clip anchoring system results in a comparatively very stable preparation for longer than 2 weeks. 


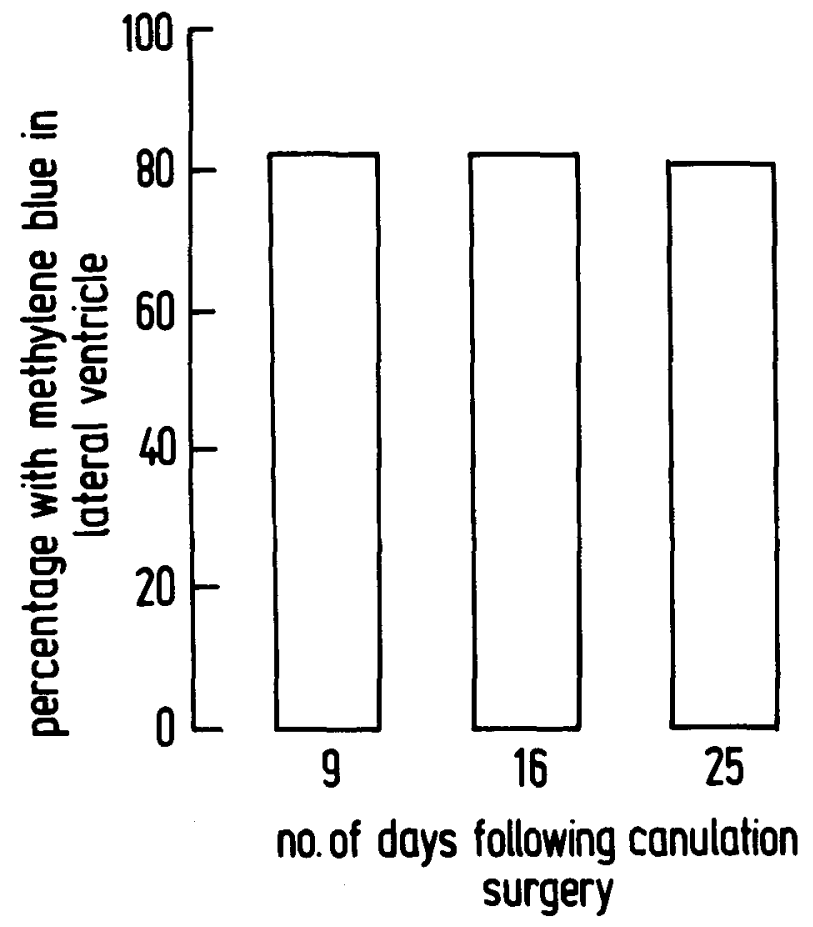

Figure 3. The "hit rate" for injection into the lateral ventricle is good and consistent over time following surgery.

common. Wound margins were well healed, with no evidence of tissue damage or infection. The cortex was damaged in the vicinity of the guide cannula, and minor gliosis was associated with the damage. Wound clip lesions were not as great as those usually encountered when skull screws are used as anchoring devices.

The use of easily available, and often disposed of, parts for the cannula construction and anchoring system makes this procedure extremely inexpensive. In our laboratory a two-person team can implant a cannula in $3 \mathrm{~min}$, an important time-conserving feature in large sample-size studies.

\section{REFERENCES}

Crane, L. A., \& Glick, S. D. Simple cannula for repeated intracranial drug administration in rats. Pharmacology, Biochemistry and Behavior, 1979, 10, 799-800.

Kokkinidis, L., Raffler, L., \& Anisman, H. Simple and compact cannula system for mice. Pharmacology, Biochemistry and Behavior, 1977, 6, 595-597.

Laird, H. E. II, Hermansen, J. E., \& Huxtable, R. J. An electrode-cannula unit for intracerebral electrical stimulation, EEG recording and drug administration in small animals. Pharmacology, Biochemistry and Behavior, 1979, 10, 429-431.

Lehmann, A. Atlas stereotaxique du cerveau de la souris. Paris: Centre National de la Recherche Scientifique, 1974.

RoutTenBe RG, A. Intracranial chemical injection and behavior: A critical review. Behavioral Biology, 1972, 7, 601-641.

Villiger, J. W., \& Chute, D. L. Papaverine facilitates passive avoidance in mice. Society for Neuroscience Abstracts, 1979 , $5,326$.

(Received for publication November 14, 1979; revision accepted April 5, 1980.) 